Markus Wessendorf

\title{
Ein kurzer Abriß der austral-asiatischen Performance
}

Anfang Mai 1998 wurde auf dem australischen Fernsehkanal Ten wiederholt der Werbespot einer Teppichkette ausgestrahit: "Machen Sie die Asienkrise zu barem Geld!" Cash in on the Asian crisisl war das schlagkräftige Motto dieses Spots, der Teppiche aus asiatischen Ländern zu Dumping-Preisen anbot und den seit dem Juli 1997 erfolgten Einbruch auf den asiatischen Finanzmärkten als günstige Gelegenheit für ein "Schnäppchen" deklarierte. Dieser Werbespot wurde zu einem Zeitpunkt ausgestrahlt, an dem die Schreckensvision einer durch den Zusammenbruch der asiatischen Finanzmărkte in Mitleidenschaft gezogenen australischen Wirtschaft bereits monatelang die Medienöffentlichkeit bestimmt hatte.

Als "gelbe Gefahr" war Asien seit jeher von vielen Australiern wahrgenommen worden: die Angst vor den auf den australischen Goldfeldern eingesetzten Chinesen als hart arbeitenden und das Lohnniveau drückenden Arbeitskräften führte Ende des 19. Jahrhunderts zum Stopp jeglicher nicht- europäischen Einwanderung; seit dem 1905 erfolgten Sieg über Rußland galt Japan als gefährliche Nation mit Expansionsgelüsten, was durch die Ereignisse des 2. Weltkrieges noch eine Steigerung erfuhr; in den 50er und 60er Jahren repräsentierten China und Vietnam dann vor allem die kommunistische Bedrohung. Mitte der sechziger Jahre wurde die "White Australia Policy" aufgegeben und erstmals die Einwanderung aus asiatischen Ländern gefördert. Seit Ende der sechziger Jahre entwickelten sich einige asiatische Nationen dann allmählich zu sogenannten kapitalistischen "Tigern". Die von mehreren Labour-Regierungen von Mitte der achtziger Jahre bis 1996 verfolgte Politik des Republikanismus, des Multikulturalismus und der gezielten Integration Australiens in den südostasiatischen Raum erfolgte dann vor allem aus wirtschaftlichen und sicherheitspolitischen Erwägungen. Meaghan Morris, Mitbegründerin der Cultural Studies in Australien, schreibt dazu:

Als die Labour-Regierung seinerzeit das republikanische Anliegen unterstützte, wurde das seit den siebziger Jahren für den heimischen Multikulturalismus typische Argument eines Management der Vielfalt mit einer 'nach außen gerichteten' Rhetorik der Werbung für diese Vielfalt kombiniert ('Australien ist eine multikulturelle Nation im asiatisch-pazifischen Raum'), und dazu benutzt, die auf Deregulierung zielende ökonomische Reform in den achtziger Jahren zu rechtfertigen. Seitdem mehr als zwei Drittel des 
australischen Handels in der Region erfolgen und sich engere politische und militärische Bindungen zu nahegelegenen Ländern (insbesondere Indonesien) herausbilden, ist es praktisch, nun eine unabhängige Identität zu etablieren. 1

Das vorerst letzte Stadium in der Entwicklung des australischen Verhältnisses zu Asien trat dann mit der asiatischen Finanzkrise im Juli 1997 ein. Zum einen wurde jetzt die "Zăhmung der Tiger" (so der Titel einer kürzlich erschienenen Publikation über das Ende des asiatischen Wirtschaftswunders²) in den Medien fast mit einer gewissen Erleichterung, nämlich als Bestätigung der ökonomischen überlegenheit Australiens als westlicher Nation über den geopolitisch zwar näher gerückten, jedoch kulturell weiterhin fernen Osten registriert, zum anderen aber gerade die vollzogene wirtschaftliche Vernetzung mit dem asiatischen Markt als Bedrohung wahrgenommen. Der erwähnte Werbeslogan, "Cash in on the Asian crisis", legte in dieser Stimmungslage die primär wirtschaftlichen Motive einer Annäherung Australiens an Asien bloB, indem er suggerierte, da $B$ der australische Konsument als Gewinner aus einer Krise hervorgehen und Profit schlagen konnte, die in keinster Weise ihn betraf, sondern sich - wie in kolonialen Zeiten - lediglich auf dem Feld des orientalischen "Anderen" ereignete.

Der vor allem in der ersten Hälfte der neunziger Jahre zu beobachtende Boom australischer Performances mit einem deutlichen Asien-Bezug war u.a. das Resultat langjähriger Bemühungen einiger aufeinanderfolgender LabourRegierungen, die Anbindung an den asiatischen Markt auch kulturell zu zementieren und die Australier dazu zu bewegen, sich eher als Teil Asiens zu begreifen als sich mit dem europäischen Erbe zu identifizieren - die geographische Lage sozusagen über die geschichtliche Herkunft zu stellen. Diese Politik führte zur Einrichtung von Förderinstitutionen wie Asialink und hatte u.a. zur Folge, daß das Performing Arts Board des Australia Council, ebenfalls in den frühen Neunzigern, die Hälfte des Budgets für internationale künstlerische Projekte in der asiatischpazifischen Region ausgab. Das Adelaide Festival von 1994 war vor allem auf Theaterproduktionen aus dem asiatischen Raum fokussiert, und Sydney und Melbourne richteten erstmals seither jährlich stattfindende asiatische Theaterfestivals aus. Erst seit der Mitte 1996 erfolgten Regierungsübernahme durch John Howards konservative Liberal Party (die bei den Wahlen im Oktober 1998 an der Macht bestätigt wurde) hat eine tendenzielle Abkehr von der multikulturellen

1Meaghan Morris. Too Soon, Too Late. Indiana University Press, 1998, 196 (Übersetzung: MW). 2siehe hierzu Robert Garran. Tigers Tamed. The End of the Asian Miracle. Sydney: Allen \& Unwin,
1998 .
Politik der Vorgänger stattgefunden, während die asiatische Finanzkrise zugleich die Rückkehr Australiens zu einer paternalistischen Haltung innerhalb der asiatisch-pazifischen Region (insbesondere Indonesien und Malaysien gegenüber) begünstigt hat.

Von politischen Interessen abgesehen, die insbesondere die Herausbildung eines institutionellen und finanziellen Rahmens ermöglicht haben, bestimmen diverse andere Faktoren das spezifische Erscheinungsbild australasiatischer Performances in den neunziger Jahren. Historisch bedeutsam ist z.B. der Einfluß, den asiatische Darstellungsformen bereits seit Mitte des 19. Jahrhunderts auf die Entwicklung des australischen Theaters ausgeübt haben. So traten chinesische Theatergruppen zwischen 1850 und 1870 - und zwar mit einer Mischung aus Akrobatik, kantonesischer Oper und Militärstücken - auf den Goldfeldern Victorias auf; japanische Gruppen gastierten dann erstmals 1867 mit Seiltanz, populären Szenen aus dem Kabuki und Schwertkămpfen. Die im Lande bleibenden Chinesen haben ihre Aufführungstradition bis in die Gegenwart hinein aufrechterhalten, wohingegen sich viele japanische Künstler den zahlreichen Zirkussen anschlossen, die in der zweiten Jahrhunderthälfte durch Australien tourten (so trat u.a. die Godayou-Familie in den neunziger Jahren mit dem Wirths' Circus und dem FitzGerald Brothers' Circus auf). Neben den vor allem aus England und Amerika stammenden Zirkusgruppen beeinflußten diese chinesischen und japanischen Künstler langfristig die Entwicklung einer körperbetonten, akrobatischen und dem Zirkus nahen Aufführungsrichtung, die noch heute einen Großteil der alternativen und insbesondere feministischen Performance-Szene in Australien kennzeichnet (u.a. Circus Oz, Vulcana, Rock'n Roll Circus). ${ }^{3}$ Seit den fünfziger Jahren dieses Jahrhunderts haben zudem Theatergruppen aus China und Japan regelmäßig in Australien gastiert: 1956 die Peking Opera Company, 1983 das Jiangsu Peking Opera Theatre und 1988 die Peking Opera Troupe of China mit einer vollständigen Aufführung von Wu Zuguangs Die drei Niederlagen des Tao Sanchun. Eine zusammenhängende japanische Kabuki-Produktion war erstmals 1978 beim Adelaide Festival zu sehen; 1982 kam Min Tanaka mit seiner Form des Butoh nach Australien, und sowohl No als auch Kyogen wurden erstmals 1986 von der Kanze-Truppe in Australien präsentiert.

Im Kontrast zur Situation z.B. in den Vereinigten Staaten, aber auch in Europa, herrscht in Australien nicht nur im journalistischen und akademischen,

${ }^{3}$ siehe hierzu Ev Beissbarth und Jo Turner ( $\mathrm{Hg}$.). Women's Circus: Leaping off the Edge. Melbourne: Spinitex, 1997 
sondern auch im künstlerisch-praktischen Bereich - und zwar als historisches und wenig problematisiertes Erbe der ehemals vorherrschenden britischen Kultur - immer noch die Tendenz vor, an einer starren Trennung zwischen den künstlerischen Gattungen festzuhalten. Dies hat u. a. zur Folge, daß die Entwicklung der aus der Bildenden Kunst hervorgegangenen australischen Performance Art seit den späten sechziger Jahren lediglich von Kunsthistorikern verfolgt und dokumentiert worden ist. So findet sich in der 1997 erschienenen Ausgabe des Concise Companion to Theatre in Australia zwar kein Eintrag unter dem Stichwort "Performance", dafür jedoch für "Performance Art" folgende Definition: "Sie kann am ehesten als Technik innerhalb der Kunstgattung Skulptur gelten. [...] Sie ist Anti-Theater, insbesondere gegen das vorherrschende Theater. [...] Performance Art versucht, eine direkte Erfahrung ohne kulturelle Konditionierung zu bieten." 4 Die Reduzierung der Performance-Kunst auf eine Technik und ihre Zuordnung zu einem Bereich der Bildenden Kunst, die Hervorhebung ihrer Anti-Theatralität als auch ihre als Intention behauptete, jegliche sprachlich-symbolische Vermittlung unterschreitende Direktheit dienen hier vor allem - per Entgegensetzung - der Festschreibung des Begriffs eines Theaters, das sich der Nähe zum Sinn/Wort/ Geist (d.h. zum logos als dem klassischen Gegensatz zur bloßen Technik = technè) sicher weiß und sich deshalb vom bloß Räumlichen, Körperhaften - nämlich der Skulptur - abhebt, den Modus seiner eigenen Theatralität nie in Frage zu stellen gezwungen ist und darüber hinaus statt einer sinnlich-taktilen eine auf Sinnstiftung abzielende Rezeptionsweise erfordert. Diese generelle Definition unterschlägt, daß sowohl Theater als auch Performance - und zwar vor allem im amerikanischen und europäischen Raum - in den vergangenen zwanzig Jahren beständig an der Auflösung der Möglichkeit einer solchen Entgegensetzung ihrer Begriffe gearbeitet haben, daß das Theater sich u. a. mit Zeitstrukturen, Raumkonzepten, der audiovisuellen Materialität der Aufführung und der In-Szene-Setzung der ihm eigenen Semiose-Prozesse auseinandergesetzt hat, während die meisten Performance-Projekte seit den späten siebziger Jahren auf vielfăltigste Weise mit Formen der Theatralităt experimentiert haben. ${ }^{5}$ Im Hinblick auf die Auseinandersetzung mit asiatischen Theaterformen kommt hinzu, daß viele

${ }^{4}$ Philip Parsons u. Victoria Chance ( $\mathrm{Hg}$.). Concise Companion to Theatre in Australia. Sydney: The Currency Press, 1997, 216 (Ubersetzung: MW).

Der erweiterte Performance-Begrift, den ich in dieser Untersuchung voraussetze, umfaßt sowohl Theater als auch Performance Art, wobel ich, wie Philip Auslander, davon ausgehe, daß Auslander. From Acting to Pertormance. Essays sei es als deren Verdrängtes (siehe Philip Auslander. From Acting to Performance. Essays in Modernism and Postmodernism. London;
New York: Routledge, 1997, 3t.). amerikanische Künstler, die in ihren Inszenierungen z.B. auf japanische Theaterformen zurückgreifen (Robert Wilson, Elizabeth LeCompte u.a.), aufgrund ihrer Prägung durch die Kunst- und Performance-Szene New Yorks nicht primär an den Schauspieltechniken, sondern der umfassenderen ästhetischen Gestaltung, der mise en scène des No oder Kabuki und der dieser jeweils zugrundeliegenden Konzepte von Handlungsdramaturgie, Raum, Zeit, Musik, Choreographie und der Erzeugung spezifischer theatraler Effekte interessiert sind. Desgleichen haben bestimmte Entwicklungen innerhalb der Künste seit den sechziger Jahren (u.a. Concept Art, Serialismus, Minimalismus, dekonstruktivistische Architektur) in der Arbeit dieser Künstler nicht nur deutliche Spuren hinterlassen, sondern wurden dort zudem hăufig in einen kontrastierenden Bezug zu asiatischen Ästhetiken gebracht. In Australien hat es hingegen vor Mitte der achtziger Jahre kaum Berührungspunkte zwischen der Bildenden Kunst, d.h. insbesondere der als Kunst verstandenen Performance Art, und dem Theater als Performing Arts gegeben - weshalb sich Ansätze zu einer konzeptionellen Hinterfragung bzw. künstlerischen Erneuerung des australischen Theaters, die den Schauspieler nicht länger als zentrale und dominierende Instanz einer linearen Handlungsdramaturgie oder, umgekehrt, als "reinen Körper" innerhalb eines von dramaturgischen Erwãgungen möglichst unbelasteten Spektakels vorsehen, erst innerhalb der letzten Dekade feststellen lassen. ${ }^{6}$

Was Performances bildender Künstler betrift, die in dem 1970 durch Mike Parr, Peter Kennedy und Tim Johnson etablierten Inhibodress-Künstleratelier in Woolloomooloo eine erste dauerhafte Aufführungsstätte fanden, war das Interesse an der asiatischen Kultur eher gering, da die Bemühungen um die Anerkennung der eigenen Arbeit innerhalb der nationalen Kunstszene sowie der Versuch, vor allem international an Kunstströmungen in den Vereinigten Staaten und Europa Anschluß zu finden, hier im Vordergrund standen. Lediglich der Weltentwurt des Zen-Buddhismus - vielleicht vermittelt durch den Einfluß von John Cage - und damit einhergehende Atem- und Körpertechniken spielten in den Performances einiger Künstler eine strukturiende Rolle. Gary Willis, der in 6Eine neuere australische Publikation, die tatsáchlich von einem erweiterten Performance-Begrift
(im Sinne Philip Auslanders) ausgeht, ist das seit 1993 alle zwei Monate erscheinende Magazin (im Sinne Philip Ausime. Australia's Innovative Arts (http://www.rtimearts.com/ opencity). Die hingegen 1998 bei Currency Press (Sydney) erschienene und von Richard James Allen und Karen Peariman herausgegebene Anthologle Performing the Unnameable ist zwar begrußenswert, da sie erstmals Auttuhrungstexte von australischen Performances der letzten Jahre versammelt und zugänglich macht, laßst aber, wie die Herausgeber hinsichtlich ihrer Auswahlkriterien betonen, Projekte der "Performance Art" explizit außen vor. 
japanischen und thailändischen Klöstern Zen studiert hatte, konzentrierte sich in Performances wie The all senses ball (Canberra 1973) und ZZZZZ (Canberra City Plaza 1973) auf funktionelle Abläufe in Arbeitsprozessen und die Verwandlung dieser alltăglichen Erfahrungen in künstlerische. ${ }^{7}$ Kevin Mortensen versuchte in seiner Performance The rocking (Canberra 1978), durch die zwangweise Lenkung seiner Konzentration auf einen forcierten Atemrhythmus die ZenTechnik zu duplizieren. Mortensen war auf einer Bahrenkonstruktion festgeschnallt, die sich ūber einer Mittelachse wie eine Wippe bewegen ließ. Die zwanzigminütige Aktion, während derer ein Assistent Mortensen in einem Rhythmus, der ungefähr dessen normaler Atmung entsprach, auf und ab schaukelte, hatte bei dem Künstler lebhafte Halluzinationen noch eine halbe Stunde nach dem Ereignis zur Folge. ${ }^{8}$

Ein wesentlicher Faktor, der die Herausbildung einer austral-asiatischen Performance-Szene in den letzten Jahren wesentlich mitgeprägt hat, bestand vor allem in dem interesse euro-australischer Theaterkünstler an asiatischen Schauspieltechniken, ein Interesse, das seit Mitte der achtziger Jahre häufig zu Studienaufenthalten in Japan, Indien oder China führte und langfristig zur Integration dieser Techniken in lokale Arbeits- und Inszenierungspraktiken beitrug. Dieses Interesse ging primär von Schauspielern aus, die sich bestimmte Körper- und Stimmtechniken aneignen wollten, um so eine physische Intensităt der Darstellung auf der Bühne zu erreichen, die sich vom vorherrschenden Bühnenrealismus angelsăchsicher Provenienz deutlich abhob. Als populärste Technik galt dabei die körperbetonte und zum Teil dem Kabuki entlehnte Methode des japanischen Regisseurs Tadeshi Suzuki, an dessen Workshops in Toga zahlreiche australische Schauspieler teilnahmen, die in ihrer späteren Arbeit die Suzuki-Technik in den Mittelpunkt stellen sollten. Sowohl der Dramatiker und Regisseur Norman Price als auch die Gruppen Sidetrack Performance Group. Frank Productions, Zen Zen Zo und die mittlerweile aufgelöste Sydney Front berufen sich auf Suzuki als Vorbild.

Angesichts der Arbeit dieser Gruppen stellt sich jedoch die Frage, auf welcher Ebene und aus welchen Motiven heraus die Auseinandersetzung mit dem asiatischen Theater erfolgt. Werden Techniken wie die Suzukis in die eigene Darstellungsweise integriert, um damit in einen konstruktiven interkulturellen

7siehe Anne Marsh. Body and Selt. Pertormance Art in Australia 1969-92. Melbourne: Oxford
University Press, 1993, 13t. University Press, 1993, $13 t$
Dialog mit der jeweiligen, in diesem Falle japanischen Theaterkultur zu treten, oder erfolgt die Übernahme einer solchen Technik lediglich aus einer orientalisti-schen Faszination heraus? Oder geht es vielmehr darum, neue Ausdrucksmittel zu erschließen, die der nach wie vor dominierenden britischen Schauspieltradition möglichst entgegengesetzt sind? Leider nimmt die fast ausschließliche Fokussierung auf die Ausdrucksmöglichkeiten des Schauspielers häufig einen Teil für das Ganze, so als folge aus der Aneignung einer "anderen" SchauspielTell fúr das Ganze, so als folge aus der Aneignung einer "anderen" Schauspieltechnik automatisch eine "andere" Dramaturgie, Inszenierungsweise und "Politik" der theatralen Repräsentation. Häufig wird die Auseinandersetzung mit Asien gerade durch die Suche nach den Wurzeln des westlichen Theaters begründet, die diesem abhanden gekommen seien, aufgrund ihrer Universalităt aber vermeintlich in anderen Kulturen wiederentdeckt werden können. So äußert sich Nigel Kellaway, Mitbegründer des von 1986 bis 1994 bestehenden Ensembles The Sydney Front, über die Beweggründe, die ihn in den achtziger Jahren als ersten Australier zu Suzukis Trainingsstätte führten: "Wir müssen nicht definieren, was Asien ist - alles kommt von den selben Wurzeln her. Shakespeare verstand das Ritual, und das asiatische Theater ist damit in steter Berührung geblieben." Inszenierungen der Sydney Front, insbesondere John Laws/Sade von 1987 und Don Juan von 1991, stellen vermittels der Auseinandersetzung mit George Batailles Theorie des Exzesses und Jean Baudrillards Schriften über die Vertühtailles Theorie des sichtlich der Körpergrenzen, der Geschlechter, aber auch der Subjekt- und Objichtlich der Korpergrenzen, der voyeuristischen Blickes dar. ${ }^{10}$ In Don Juan wird Suzukis Körpertechnik in einer choreographischen Sequenz eingesetzt, in der sechs Darsteller (drei Männer, drei Frauen) in weißen Frauengewändern, mit weit geöffneten Beinen und tief gebeugten Knien, rhythmische Vorwärtsbewegungen machen und dabei stampfende Geräusche mit ihren Füßen erzeugen. Sowohl die weiß geschminkten Gesichter (mit rot hervorgehobenem Mund) als auch die Tatsache, daß die männlichen Schauspieler Frauenkostüme tragen, legen zudem die Anlehnung an das japanische Kabuki und seine onnagata-Tradition des männlichen Frauendarstellers nahe (wenn auch nicht ausschließlich, da des männlichen Frauendarstellers nahe (wenn auch nicht ausschließlich, da beides auch auf europäische Darstellungstraditionen zurückbezogen werden
kann). Da sowohl Suzukis Körpertechnik als auch das Make-up der Schauspieler

Tit in: Alison Broinowski. The Yellow Lady. Australian impressions or Asia. Melbourne: Oxtord University Press. 1992,139 (Übersetzung: MW).
10 siehe Jane Goodall. "Seduction and The Sydney Front, in Canadian Theatre Review 74, Frühling 1993. 32 . 
vor allem als szenische Mittel eingesetzt werden, um eine erotische und exotische Atmosphäre der Verführung herzustellen, scheint sich die Inszenierung eher unkritisch in die lange Geschichte der orientalistischen Vereinnahmung östlicher Zeichensysteme auf westlichen Bühnen einzureihen."

Bei der aus Brisbane stammenden Gruppe Zen Zen Zo wiederum stellt sich das Problem, daß theatrale Ausdrucksmittel Japans übernommen werden, ohne jedoch mehr zu sein als ein Ersatz für den Mangel eigener theatraler Konzepte und Ideen. Die Ausdrucksmittel werden dekontextualisiert, aber nicht erneut dramaturgisch rekontextualisiert. Das führt u.a. zu explizit als "Butoh" deklarierten Aufführungen Zen Zen Zos (z.B. in Steel Flesh vom September 1998, Brisbane Festival), die die asymmetrischen Körperhaltungen, verzerrten Gesichtsmasken und starren Tableaus dieser Form übernehmen, ohne daß die Dringlichkeit einer solcherart gesteigerten Expressivităt, wie sie im Butoh vor allem aus dem durch Hiroshima ausgelösten Trauma resultierte, sichtbar würde. Zudem bedienen sich die von Suzuki inspirierten Inszenierungen Zen Zen Zos nicht nur dessen Körper- und Schauspieltechnik, sondern übernehmen - auch hier auf den "Suzuki-Effekt" setzend - Stũcke in ihren Spielplan, die der japanische Regisseur selbst zuvor erfolgreich szenisch umgesetzt hat, so z.B. Macbeth und Die Bakchen.

Suzuki selbst nahm 1992 die Einladung des Playbox Theatre in Melbourne an, seine Macbeth-Bearbeitung (die er zuvor bereits in Japan inszeniert hatte) mit australischen Schauspielern zu realisieren. Diese Zusammenarbeit kam aufgrund der jahrelangen Bemūhungen des kūnstlerischen Leiters des Playbox Theatre zustande, der sich von der Kooperation Suzukis mit australischen Schauspielern eine theatralisch fruchtbare, interkulturelle Begegnung erhofft hatte. Die Inszenierung kam jedoch bei der Kritik nur mäßig an, und die Arbeitsweise des Regisseurs wurde im nachhinein von beteiligten Darstellern vehement kritisiert, da Suzuki weniger an gegenseitiger kultureller Verständigung und Zusammenarbeit als vielmehr an der Disziplinierung der Schauspieler im Sinne seiner eigenen Technik interessiert gewesen sei (so hatte Suzuki angeblich während der Proben behauptet, australische Schauspieler seien faul'12). Die nicht eingelösten Erwartungen und kulturellen Mißverständnisse, die der aus australi-

"1siehe hierzu das Kapitel "Orientalism in the theatre" in: John M. MacKenzie. Orientalism. History Theory and the Arts. Manchester University Press, 1995, 176-207.

in Aboutphy. 'Operation Hypothesis: Tadashi Suzuki's 'Toil and Trouble' tour, Australia 1992*: In About Pertormance. Pertormances East/West. Centre for Performance Studies/University of Sydney, Working Papers 2, 1996, 44 scher Sicht so enttäuschenden Kooperation offensichtlich zugrundelagen, drängen auch hier die Frage auf, wer und aus welchen Gründen ein Interesse an drangen auter mentiert, daß Suzukis Interesse an dem Projekt eher politisch als künstlerisch motiviert gewesen sei, da es diesem die Möglichkeit gegeben habe, eine Produktion, die in Japan bereits erfolgreich gewesen war, ohne Risiko für seine künstletion, die in Japan bereits erfolgreich gewesen war, ohne Risiko aus a selischen Raum vorrische Reputation als Re-Inszenierung erstmals auch im australischen Raum vor-
zustellen. Playbox habe umgekehrt vor allem auf der Ebene der Public Relations zustellen. Playbox habe umgenen Projekt profitiert. ${ }^{13}$

Im Kontrast zum vermeintlichen Desinteresse Suzukis an den BesonderIm Kontrast zum Theaters hebt Keiji Sawada in einer Publikation des Japan Cultural Centre in Sydney das in den letzten Jahren gestiegene Interesse Japan Cultural Centre in Sydne der australischen Kultur hervor, was er u.a. der japanischen Bevölkerung an der australischen Kultur hervor, was er U.a. auch auf die seit 1993 in Japan bestehende Australlan Drane Ttück, John Romezurückłührt. ${ }^{14}$ Das erste innerhalb dieser Reihe erschienene Stack, John Aomdrils The Floating World1's, wurde 1995 von Makoto Sato, einem der bedeutendsten japanischen Regisseure und Dramatiker, inszeniert und mit Isao Natsuyagi, einem beliebten Filmschauspieler, in der Hauptrolle besetzt. Zur Zeit der "neuen einem bele a tratischen Theaters in den frühen siebziger Jahren entstanden Welle" des australischen Theaters in den frahen siebzige Jahren sich australigehört The Floating World zu einer Reihe von Stūcken, in denen sich australische Dramatiker europäischer Herkunft in den letzten zwei Jahrzehnten mit Asien auseinandergesetzt haben, so u.a. mit Japan während des 2. Weltkriegs (Jill Shearers Shimada, 1987), der australischen Beteiligung am Vietnam-Krieg und dearen Implikationen (Rob Georges Sandy Lee Live at Nui Dat, 1981), aber auch dem australischen Sex-Tourismus auf den Philippinen (Michael Gurrs Sex Diary of an Infidel, 1992). Les Harding, die Hauptfigur in Romerils The Floating World, wird von seinen Erinnerungen an die japanische Kriegsgefangenschaft während des 2 . Weltkriegs und die brutalen Bedingungen des Arbeitseinsatzes an der Eisenbahnlinie Burma-Thailand Jahre später während einer Kreuzfahrt mit seiner Eisenbahnlinie Burma-Thailand Jach eingeholt. Die Dramaturgie von kurzen Szenen, fragmentarischen Monologen, Vaudeville-Sketchen und Witzen reflektiert nen, fragmentarisch Hardings in einen prekären Geisteszustand, in dem sich die

13Michael Cohen. "Seventeen Stories about Interculturalism and Tadashi Suzuki", in About Performance. Performances East/West, 55. 14Keiji Sawada. Future Directions in Australia Japan Theatrica

Sydney: The Japan Foundation Papers, Ny: Currency Press, 1975. 
Anwesenden an Bord zunehmend in Gefährten und Peiniger des japanischen Kriegsgefangenenlagers verwandeln. Sato setzte in seiner Inszenierung die Szenen, in denen ein Komiker der Abendgesellschaft an Bord anti-japanische Witze erzăhit, als Puppenspiel um. Dadurch gelang es ihm, die für ein japanisches Publikum vielleicht beleidigenden Äußerungen so zu verfremden, daß die Intention des Dramatikers deutlich wurde, in Australien gängigemden, daß dia Ressentiments durch ihre Zitiorung Die Inszenierung wurde sowohl auf dent zu affirmieren, sondern bloßzustellen. ing Arts als auch auf don International Festival of Performund zwar im Doppelprem Melbourne International Festival of the Arts aufgeführt Tanakas The Head of Atombombenabur bourne, Regie: Aubrey Mellor).

Als weiterer Faktor der austral-asiatischen Performance machte sich in den achtziger Jahren erstmals auch die seit den sechziger Jahren erfolgte asiatische Einwanderung künstlerisch bemerkbar. Nach Australien eingewanderte Asiaten bzw. Ausíralier asiatischer Herkunft begannen, durch eigene Projekte aktiv an der Gestaltung eines integrativen und multikulturellen austral-asiatischen Theaters mitzuwirken. So arbeitet der aus Malaysien stammende Chandrabhanu mit seiner 1987 in Melbourne gegründeten Bharatam Dance Company an der Entwicklung einer hybriden Darstellungsform, die die klassischen indischen Tanzformen Bharata Natyam und Odissi durch Amalgamierung mit bekannteren Erzählhandlungen - z.B. aus der griechischen Tragödie - ach mit bekannteren ErPublikum zugănglich mas der griechischen Tragodie - auch einem australischen kumar versucht mit ihrer eben soll. Die seit 1985 in Australien lebende Tara RajDance Company etablieren, indem hingegen, die indische Tanzform Mohiniattam in Australien zu end Finfur sit vertrauteren Formen auf begleitende Einführungsvortrăge und -Kurse setzt. In Brisbane versucht Indija Mahjoeddin, Australierin indonesischer Herkunft, die volkstūmliche, aus dem Kamptsport stammende indonesische Theaterform Randai mit australischen Musikern und Darstellern zu realisieren. Ihre 1997 am Princess Theatre in Brisbane autge führte Inszenierung The Horned Matriarch - The Story of Reno Nilam basierte au einem Stûcktext, der sich vor allem auf Motive aus der indonesischen MinangKultur bezog. Das von Mahjoeddin, Larisa Chen und Jilli Streit gemeinsam entwickelte und im Februar 1998 am Brisbane Ethnic Music and Arts Centre urautgeführte Projekt Imagining Mata Hari stellte hingegen den Versuch dar, durch eine Kombination asiatischer Darstellungstechniken und die Auseinandersetzung mit Mata Hari als einer exemplarischen Verkörperung des Orientalismus auf westlichen Bühnen diesen in seiner Grundstruktur szenisch zu analysieren. Die meisten dieser Projekte zeichnen sich jedoch, wie viele Versuche, traditionelle und streng kodierte asiatische Formen durch Hybridisierung in einen anderen Kontext übersetzen zu wollen, durch einen prekären Status aus, den die postkoIoniale Kritikerin Purushottama Bilimoria dadurch charakterisiert hat, "daß sie, obwohl sie aufregend neuartig und unterhaltend sind, in den Spalt zwischen traditioneller und innovativer Arbeit geraten und letztlich als Amalgam verschiedener kultureller Hervorbringungen und kūnstlerischer Techniken ohne kohărenten Zusammenhalt wahrgenommen werden." 18

In den neunziger Jahren haben australische Künstler asiatischer Herkunft eine Form des autobiographischen Solo-Projekts entwickelt, die explizit die Einwanderungserfahrung bzw. den hybriden sozialen Status zwischen zwei Kulturen reflektiert, ohne einer traditionellen Darstellungsform verpflichtet zu sein. Als einer der wichtigsten australischen Performance-Künstler der letzten Jahre gilt in dieser Hinsicht der zuvor bereits als Fotograf bekannt gewordene William Yang. Yang, der 1969 zu den Gründungsmitgliedern des Performance Syndicate in Sydney gehörte und für diese Gruppe Stūcke schrieb, trat 1985 mit The Face of Buddha im Belvoir Street Theatre in Sydney auf, dem ersten einer Reihe von Monologen im Vortragsstil einer Diavortührung. In Sadness (1992) ${ }^{17}$ überkreuzen sich zwei fotodokumentarisch begleitete Erzählstrănge: die Geschichte der an AIDS verstorbenen Freunde und die Geschichte seiner Familie, die Ende des 19. Jahrhunderts von China nach Queensland ausgewandert ist - Erzählstränge, die durch die Reflexion Yangs aut sein doppeltes Außenseitertum in der australischen Gesellschaft als Homosexueller und als Australier chinesischer Herkunft verklammert sind.

Yang vergleichbar setzt sich Anna Yen in ihrer 1997 in Brisbane aufgeführten Performance Chinese Take Away mit der Geschichte ihrer Großmutter während der dreißiger Jahre in China und Hongkong auseinander, greift dabei jedoch auf ein Ausdrucksrepertoire zurück, das sich aus Zirkusakrobatik, Tai Chi, Kampfsport und Feldenkrais-Technik zusammensetzt.

16Purushottama Bilimoria. "Traditions and transition in South Asian performing arts in multicultural Australia*, in Culture, Difference and the Arts. Hg. v. Sneja Gunew u. Fazal Rizvi. Sydney: Allen \& Unwin, 1994, 121 (übersetzung: MW)

17 William Yang. Sadness. Sydney: Allen \& Unwin, 1996 
Hung Le hingegen thematisiert in seinem Programm Now and Zen (Adelaide Fringe Festival, 1998) seine Kindheit als vietnamesischer Flüchtling im xenophobischen Australien, wobei er die Rolle des anglo-amerikanischen standup comedian mit der des ocker - dem populären Typus des australischen "bushman" - amalgamiert und dabei versucht, die durch diese Position implizierte Komplizenschaft mit einem weißen, männlichen Publikum durch anti-vietnamesische Witze sowohl zu verfremden als auch zugleich in ihrer problematischen Ausschlußfunktion transparent zu machen (der andere, über den man lacht, hat hier die Rolle des Gleichgesinnten okkupiert, der den Witz erzählt). ${ }^{18}$

In der Debatte um die "Asiatisierung" Australiens steht die Frage nach der nationalen und kulturellen Identităt auf dem Spiel. In kaum einer Medienőffentlichkeit des Westens ist genau die Frage dieser Zugehörigkeit zum Westen ein derartiges Dauerthema wie in Australien. Der australische Kunstkritiker Paul Foss argumentiert in seinem einflußreichen (und selbst von Baudrillard beeinflußten) Essay "Theatrum Nondum Cognitorum", daß die Geschichte Australiens aus europäischer Perspektive vor allem von Wunsch- und kartographischen Projektionen als auch imaginären Kartographien eröffnet wurde, die der britischen Besiedlung bis zu 2000 Jahre vorausgingen. Die Suche nach den Antipoden trieb seit jeher die europăische Expansion voran, als strukturelle Umkehr alles dessen, was die Lebensbedingungen in Europa - nicht nur răumlich - einzuschränken schien. Die Antipoden als Nicht-Ort, als unmöglicher Ort zugleich der Fülle wie des Vakuums, eröffnen fûr Foss den Schauplatz, an dem sich die Geschichte Australiens entfaltet: als ein Theatrum Nondum Cognitorum", ein "Theater des noch nicht Entdeckten", das bis heute den längst vermessenen und besiedelten Raum Australiens mit der "ătherischen und traumhaften Eigenschaft des nevernever" 20 heimsucht. Ist eine austral-asiatiische Performance möglich, die nicht länger Resultat einer europäischen Projektion wăre? Würde die Performance eines "asiatisierten" Australiens nicht erst recht einer europäischen Projektion (der des "Anderen" down under) genügen? Die austral-asiatische Performance der neunziger Jahre findet statt in der als "Theatrum Nondum Cognitorum" bezeichneten Kluft, einem aufgeschobenen Nationaltheater als Theater der aufgeschobenen nationalen Identităt: zwischen Monarchie und Republik, britischer

${ }^{18}$ siehe Tseen-Ling Khoo. "The ocker ethnic: Reading Hung Le and cultural identity", in: Postscript. The Journal of the English Postgraduate Society. University of Queensland. Herbst $1998,24-28$.

19 siehe Paul Foss. "Theatrum Nondum Cognitorum [1981]", in: What is Appropriation? Hg. v. Rex

Butler. Brisbane/Sydney, 1996, 125.
20 ebd., 127 (Ubersetzung: MW).
Tradition und amerikanischer Hegemonie, West und Ost, Subjekt und Objekt des Kolonialismus, weißen Besitzständen und Landrechten der Aborigines, zwischen der Zweihundertjahrfeier der weißen Besiedlung 1988 und dem hundertjährigen Bestehen der Föderation im Jahre 2001. 